\title{
Cold cathode emission studies on topographically modified few layer and single layer $\mathrm{MoS}_{2}$ films
}

\author{
Anand P. S. Gaur, ${ }^{1}$ Satyaprakash Sahoo,,${ }^{1,2, a), b)}$ Frank Mendoza, ${ }^{1}$ Adriana M. Rivera, ${ }^{1}$ \\ Mohit Kumar, ${ }^{2}$ Saroj P. Dash, ${ }^{3}$ Gerardo Morell, ${ }^{1}$ and Ram S. Katiyar ${ }^{1, b)}$ \\ ${ }^{1}$ Department of Physics and Institute for Functional Nanomaterials, University of Puerto Rico, San Juan, \\ Puerto Rico 00931, USA \\ ${ }^{2}$ Institute of Physics, Bhubaneswar 751005, Odisha, India \\ ${ }^{3}$ Department of Microtechnology and Nanoscience, Chalmers University of Technology, SE-41296, \\ Göteborg, Sweden
}

(Received 27 August 2015; accepted 5 January 2016; published online 26 January 2016)

\begin{abstract}
Nanostructured materials, such as carbon nanotubes, are excellent cold cathode emitters. Here, we report comparative field emission (FE) studies on topographically tailored few layer $\mathrm{MoS}_{2}$ films consisting of $\langle 0001\rangle$ plane perpendicular $(\perp)$ to $c$-axis (i.e., edge terminated vertically aligned) along with planar few layer and monolayer (1L) $\mathrm{MoS}_{2}$ films. FE measurements exhibited lower turn-on field $E_{\text {to }}$ (defined as required applied electric field to emit current density of $10 \mu \mathrm{A} / \mathrm{cm}^{2}$ ) $\sim 4.5 \mathrm{~V} / \mu \mathrm{m}$ and higher current density $\sim 1 \mathrm{~mA} / \mathrm{cm}^{2}$, for edge terminated vertically aligned (ETVA) $\mathrm{MoS}_{2}$ films. However, $\mathrm{E}_{\mathrm{to}}$ magnitude for planar few layer and $1 \mathrm{~L} \mathrm{MoS}_{2}$ films increased further to 5.7 and $11 \mathrm{~V} / \mu \mathrm{m}$, respectively, with one order decrease in emission current density. The observed differences in emission behavior, particularly for ETVA $\mathrm{MoS}_{2}$ is attributed to the high value of geometrical field enhancement factor $(\beta)$, found to be $\sim 1064$, resulting from the large confinement of localized electric field at edge exposed nanograins. Emission behavior of planar few layers and $1 \mathrm{~L}$ $\mathrm{MoS}_{2}$ films are explained under a two step emission mechanism. Our studies suggest that with further tailoring the microstructure of ultra thin ETVA $\mathrm{MoS}_{2}$ films would result in elegant FE properties. (C) 2016 AIP Publishing LLC. [http://dx.doi.org/10.1063/1.4940306]
\end{abstract}

The cold cathode emitters based technologies, such as flat displays, ${ }^{1}$ vacuum microelectronics, ${ }^{2}$ x-ray sources, ${ }^{3,4}$ and electron guns, ${ }^{5}$ requisite coherent electron emission and high current density at low turn-on field $\left(\mathrm{E}_{\mathrm{to}}\right)$. For this purpose, the best-preferred metal emitters are engineered in the form of a sharp pointed ${ }^{6}$ source to subdue $E_{t o}$ further. In such emitters, due to the presence of the large gradient of localized electric field at the pointed/sharp surface, there is a significant improvement in the emission current density. Analogous to this, materials with tailored structures, such as tubes, ${ }^{7-9}$ sheets, ${ }^{10}$ ribbons, ${ }^{11}$ and wires, ${ }^{12,13}$ also exhibit elegant field emission (FE) behavior. For example, field emitters consisting of the vertically aligned carbon nanotubes (CNTs) array have recently been exploited in microwave $\operatorname{diode}^{2}$ and electron gun ${ }^{5}$ applications. Along with CNT other carbon nanomaterials, such as graphene, nanodiamonds, and nanoribbons, were also studied for cold cathode emission. Atomically thin, two-dimensional (2D) monolayer graphene has many novel properties, ${ }^{14,15}$ but contrary to the CNT, magnitude of $\mathrm{E}_{\mathrm{to}}$ for monolayer graphene was found to be quite high, and therefore potentially hinders its FE application. However, studies on the edges of graphene sheet (with exposed $\mathrm{C}$ atoms having incomplete $\mathrm{C}-\mathrm{C}$ bonds) have shown enhanced FE behavior with extremely low $\mathrm{E}_{\mathrm{to}}$, which is ascribed to the lower work function of edges, ${ }^{16}$ and similar observations were reported for graphene flakes ${ }^{17}$ and doped graphene. ${ }^{18}$

\footnotetext{
a) Present address: Institute of Physics, Bhubaneswar 751005, India

${ }^{b)}$ Authors to whom correspondence should be addressed. Electronic addresses: rkatiyar@hpcf.upr.edu and satya504@gmail.com
}

Recently, other 2D materials, particularly the transition metal dichalcogenides (TMD) family, have received much attention owing to their thickness dependent wide range of electrical, electronic, and optoelectronic properties. Among these, monolayer (1L) $\mathrm{MoS}_{2}$ has triggered much attention for its exciting physical properties. ${ }^{19-22}$ The anisotropy in chemical bonding in $\mathrm{MoS}_{2}$ sheets results in active edges similar to graphene sheet edges. In recent developments, synthesis of large area $\mathrm{MoS}_{2}$ film with edge terminated vertically aligned (ETVA) layers via sulfurization of molybdenum film in sulfur environment ${ }^{23,24}$ has been reported. However, unlike carbon materials, $\mathrm{MoS}_{2}$ has not been explored much for FE.

In the present work, we focus on the FE properties of few layers of planar, ETVA, and monolayer $\mathrm{MoS}_{2}$ films. Our studies revealed that the $\mathrm{MoS}_{2}$ films containing ETVA layers exhibit lower $\mathrm{E}_{\mathrm{to}}$ and higher geometrical field enhancement factor $(\beta)$ than planar few layer and monolayer $\mathrm{MoS}_{2}$ films. For $1 \mathrm{~L}-\mathrm{MoS}_{2}$, turn on field was even more than that of planar few-layer $\mathrm{MoS}_{2}$ films. FE behavior for ETVA $\mathrm{MoS}_{2}$ films was correlated to its microstructure and the emission behavior for planar few layer and $1 \mathrm{~L}-\mathrm{MoS}_{2}$ have been discussed under a serial two step emission process.

Synthesis of large area growth of ultrathin $(\sim 4-6 \mathrm{~nm})$ planar and edge oriented, as well as monolayer $\mathrm{MoS}_{2}$ films, has been reported earlier. ${ }^{25}$ In brief, $1 \mathrm{~L}$ and planar $\mathrm{MoS}_{2}$ films were synthesized on $\mathrm{SiO}_{2} / \mathrm{Si}$ substrates via chemical vapor deposition (CVD) and edge exposed $\mathrm{MoS}_{2}$ films were synthesized by sulfurization of molybdenum coated $\mathrm{SiO}_{2} / \mathrm{Si}$ substrates at two different temperatures given as $900^{\circ} \mathrm{C}$ and $550{ }^{\circ} \mathrm{C}$, respectively, in $\mathrm{H}_{2}+\mathrm{Ar}$ atmosphere. Thickness and 
microstructure of the few layer films were characterized in tapping mode atomic force microscopy (AFM-VEECO) and High Resolution Transmission Electron Microscopy (HRTEM JEOL JEM-2200FS) operated at $200 \mathrm{kV}$, respectively. Raman and photoluminescence (PL) spectroscopy were carried out using a Horiba-Jobin T64000 (triple mode subtractive) microRaman system in backscattering configuration utilizing an Argon ion laser $(514.5 \mathrm{~nm}$ line as excitation source) for phase and $1 \mathrm{~L}$ thickness confirmation of the grown $\mathrm{MoS}_{2}$ films. Prior to $\mathrm{FE}$ measurements, $\mathrm{MoS}_{2}$ films were transferred upon $\mathrm{Pt} / \mathrm{Si}$ substrates by a wet chemical etching method reported elsewhere. ${ }^{26}$ Field current was measured for all $\mathrm{MoS}_{2}$ films in a diode configuration at pressure $1.5 \times 10^{-7} \mathrm{~m}$-bar in a custom made system. ${ }^{27}$ A Stanford Research Systems PS350 and Keithley 6517A electrometer were used as the voltage source and current measurement, respectively, and emission current magnitude lower than $1 \times 10^{-12} \mathrm{~A}$ was considered at the background noise, while $E_{t o}$ was defined as the required magnitude of applied electric field necessary to emit a current density of $10 \mu \mathrm{A} / \mathrm{cm}^{2}$ following earlier reports on $\mathrm{MoS}_{2}{ }^{28,29}$ However, in a few reports on carbon-based materials, ${ }^{30} \mathrm{E}_{\text {to }}$ was defined as to achieve emission current density of $1 \mathrm{nA} / \mathrm{cm}^{2}$. To make meaningful comparisons with previous $\mathrm{MoS}_{2}$ reports, we stick to $10 \mu \mathrm{A} / \mathrm{cm}^{2}$ as $\mathrm{E}_{\mathrm{to}}$. The surface morphology and kelvin probe force microscopy (KPFM) of all layers were examined by ex-situ AFM (MFP-3D, Asylum Research) in lift mode using conductive Pt-coated tips (AC240TM, Electric-Lever, Olympus, with radius of curvature $\sim 30 \mathrm{~nm}$, stiffness $\sim 2 \mathrm{~N} \mathrm{~m}^{-1}$, and a resonance frequency of $\sim 70 \mathrm{kHz}$ ).

Figure 1(a) shows the HRTEM image of few-layer $\mathrm{MoS}_{2}$ film grown at low $\left(500^{\circ} \mathrm{C}\right)$ temperature. The microstructure exhibits distinct stripe-like features [enlarged image shown in Fig. 1(b)], distributed equally with flat, i.e., $c$-axis oriented nanocrystalline regions. The measured separation between two such stripes was found to be $\sim 0.7 \mathrm{~nm}$, which is the thickness of single layer $\mathrm{MoS}_{2}$. This thickness corroborated that striped grains consist of vertically oriented ( $\perp c$-axis) layers of $\mathrm{MoS}_{2}$. The measured film thickness and roughness were found to be $\sim 7-8 \mathrm{~nm}$ and below $1 \mathrm{~nm}$, respectively. ${ }^{44}$ In contrast to edge exposed $\mathrm{MoS}_{2}$ films, the $\mathrm{MoS}_{2}$ films grown at higher temperature (at $900{ }^{\circ} \mathrm{C}$ ) were well crystalline and contain only $c$ axis oriented planes; this we have demonstrated in our earlier reports. ${ }^{25}$ The difference in the microstructure between these two films explained under reaction kinematics has been reported elsewhere. ${ }^{25}$

Raman spectroscopy has been used to quantify different physical properties in $\mathrm{MoS}_{2}$ such as the number of layers, ${ }^{31}$

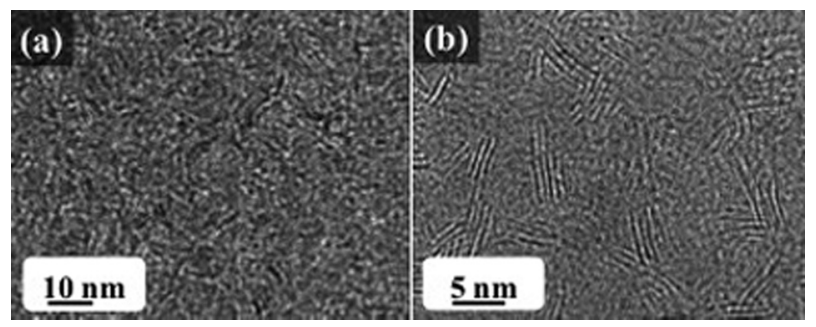

FIG. 1. (a) HRTEM image of ETVA $\mathrm{MoS}_{2}$ film. (b) Magnified image showing clear vertically aligned layers. thermal conductivity, ${ }^{32}$ crystallite size,${ }^{24}$ and doping concentration. ${ }^{33}$ Figure 2(a) shows the room temperature Raman spectra of 1L, ETVA, and few-layer $\mathrm{MoS}_{2}$ films. It can be seen from the figure that, for $1 \mathrm{~L}-\mathrm{MoS}_{2}, \mathrm{E}_{2 \mathrm{~g}}^{1}, \mathrm{~A}_{1 \mathrm{~g}}$ modes blue and red shifted, respectively, with respect to their bulk frequencies, which will be discussed later. Broadening of phonon lineshape of these modes in ETVA $\mathrm{MoS}_{2}$ film with respect to bulk $\mathrm{MoS}_{2}$ film was noticed. Raman lineshape is very sensitive to crystallite size ${ }^{34}$ and lattice disorder ${ }^{35}$ and in nanomaterials due to phonon confinement and defects the phonon line shape exhibits asymmetry and broadening.

The observed broadening and line shape asymmetry in Raman bands in ETVA MoS 2 film can be ascribed to the presence of striped and $c$-axis oriented nanocrystalline grains. Moreover, the shift in frequency of these modes in $1 \mathrm{~L}-\mathrm{MoS}_{2}$ with respect to few layer films is due to the alteration in in-plane bonding and Coulombic interaction between consecutive layers. In fact, the shift in frequency of these two modes with respect to bulk $\mathrm{MoS}_{2}$ is used to determine the monolayer nature of $\mathrm{MoS}_{2}$ and found to be in accordance with earlier reported results. ${ }^{31}$ Further, the emergence of PL in $\mathrm{MoS}_{2}$ has been used as the signature ${ }^{36}$ of its $1 \mathrm{~L}$ form. In our case, we obtained huge room temperature PL intensity, centered around $1.8 \mathrm{eV}$, which is shown in Fig. 2(b). This also confirms our $\mathrm{MoS}_{2}$ thin film to be $1 \mathrm{~L}$. For comparison, PL spectra for fewlayer $\mathrm{MoS}_{2}$ is also shown in the figure. In contrast, there is no signature of a PL band in the few layer sample indicating its indirect band gap nature.

There are few reports on FE studies on chemically synthesized $\mathrm{MoS}_{2}$ flakes $^{28}$ and flowers ${ }^{29}$ like nanostructures. The reported $\mathrm{E}_{\text {to }}$ values were found to be lower in sheets, $\sim 3.5 \mathrm{~V} / \mu \mathrm{m}$, while in nanoflowers, a high magnitude of emission current density $\sim 10 \mathrm{~mA} / \mathrm{cm}^{2}$ at higher magnitude of electric field $\sim 8 \mathrm{~V} / \mu \mathrm{m}$ was reported. The demonstrated lower magnitude of $\mathrm{E}_{\mathrm{to}}$ and high emission current density in flakes and nano flowers, respectively, were explained by considering the fact that the edges of $\mathrm{MoS}_{2}$ account for the multifold increase in the localized electric field, and this resulted in higher emission current density. However, no such
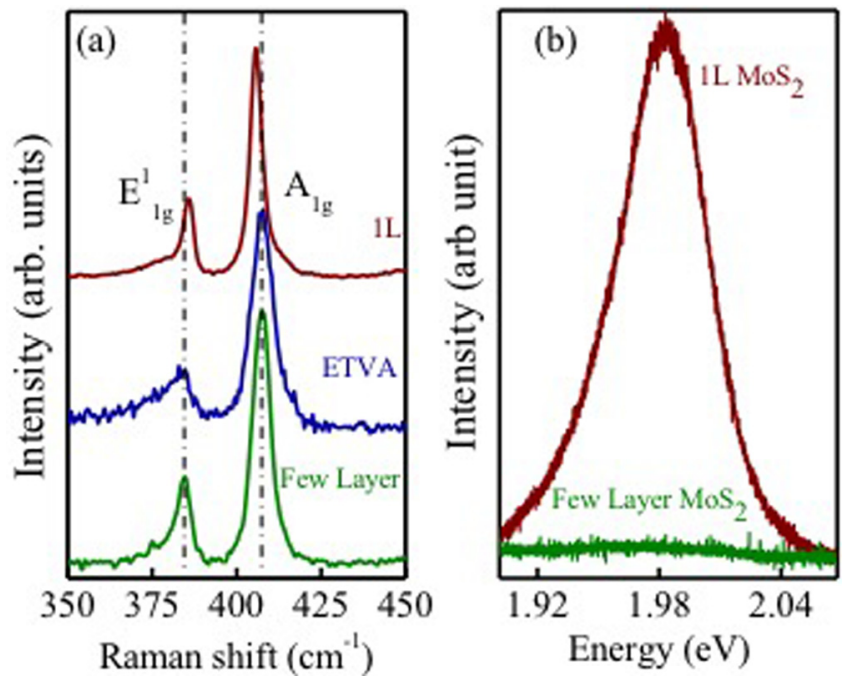

FIG. 2. (a) Raman spectrum of 1L, ETVA, and few layer $\mathrm{MoS}_{2}$ film. (b) Photoluminescence spectrum of $1 \mathrm{~L}$ and few layer $\mathrm{MoS}_{2}$. 
attempts have been made to understand the FE behavior in CVD grown large area $\mathrm{MoS}_{2}$ films. In particular, thickness dependent FE studies are still lacking. Figure 3(a) represents the FE characteristic for few layer and $1 \mathrm{~L}-\mathrm{MoS}_{2}$ films. These experimental results ascertained that $1 \mathrm{~L}-\mathrm{MoS}_{2}$ has higher turn-on field $\sim 11 \mathrm{~V} / \mu \mathrm{m}$ over the few layer form, which showed $\mathrm{E}_{\text {to }} \sim 5.7 \mathrm{~V} / \mu \mathrm{m}$. In general, Fowler-Nordheim (FN) theory, developed for metallic surface, has been used to analyze the electron emission. Accordingly, the relation for electron emission is $\mathrm{I} \propto \mathrm{AE}_{\text {eff }}^{2} \exp \left(\frac{-\mathrm{B}}{\mathrm{E}_{\text {eff }}}\right)$, where $\mathrm{A}$ and $\mathrm{B}$ are constants and $\mathrm{E}_{\text {eff }}$ is the localized electric field. Although in the case of semiconductor materials, Stratton ${ }^{37}$ discussed the effect of surface states and lowering of the conduction band due to penetration of external field. But the implication of this mathematical expression for emission current density for real semiconductor material, particularly for mono or few-layer $\mathrm{MoS}_{2}$, would be complicated and beyond the scope of the present study. In view of this, we analyze our results using Fowler-Nordheim formalism. $J / E^{2}$ versus $1 / E$ plots shown in Fig. 3(b) for fewlayer and $1 \mathrm{~L}-\mathrm{MoS}_{2}$ films exhibit linear behavior with a negative slope for higher applied fields indicating the F-N type tunneling mechanism. In such cases, a rough approximated geometrical factor $\beta$ for $1 \mathrm{~L}$ and few- layer $\mathrm{MoS}_{2}$ films can be obtained using the following equation: ${ }^{28}$

$$
\beta=\left(6.8 \times 10^{3} \times \phi^{\frac{3}{2}}\right) / \mathrm{m},
$$

where $m$ is the slope of fitted straight line and $\phi$ is work function. The estimated values of $\beta$ were found to be 601 and 503 for few-layer and monolayer $\mathrm{MoS}_{2}$ films, respectively, and are comparably low with the values for sheets and flowers. The higher magnitude of $\mathrm{E}_{\mathrm{to}}$ and lower values of $\beta$ in the present work further corroborated the smooth planar surface of $1 \mathrm{~L}$ and few-layer $\mathrm{MoS}_{2}$ films as planar geometry is not suitable to achieve a high geometrical enhancement factor. Further, the observed deviation from linear FN behavior in the $\mathrm{J} / \mathrm{E}^{2}$ versus $1 / \mathrm{E}$ plot in Fig. 3(b) at higher bias voltages is indicative of the semiconducting behavior of $\mathrm{MoS}_{2}$ films.

In the present study, the observed differences in FE characteristics between the few-layer and single layer $\mathrm{MoS}_{2}$
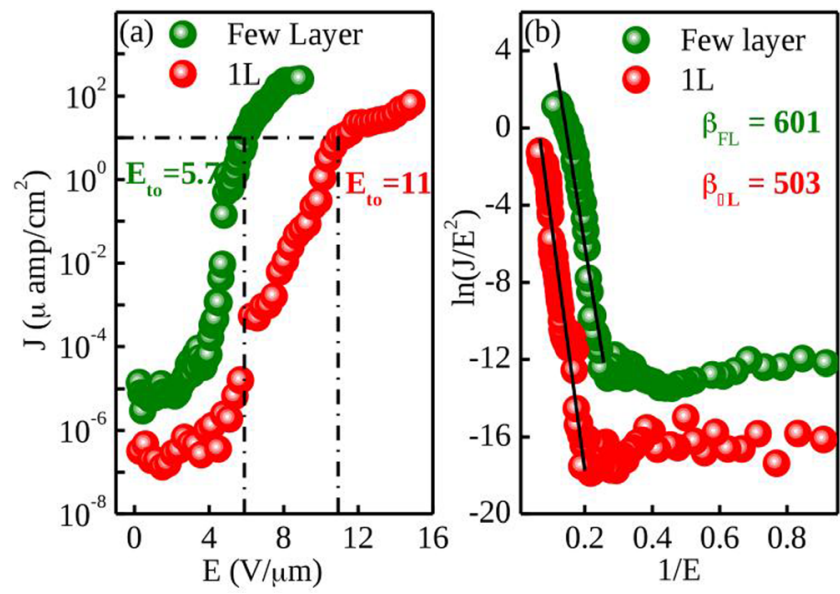

FIG. 3. (a) Field emission J-E curves. (b) FN plots for few-layer and $1 \mathrm{~L}-\mathrm{MoS}_{2}$ films. Emission current was recorded keeping the detector at $100 \mu \mathrm{m}$ away from emitting surface. films can be described under two step electron emission mechanism first proposed by Binh et al., for planar ultrathin $(\sim 5 \mathrm{~nm})$ film of wide band gap n-type semiconductor. ${ }^{38,39}$ In the first step, the electrons begin to inject into the semiconductor from the metal contact (in present case Pt) under the influence of applied external field, resulting in the space charge accumulation at the metal-semiconductor interface. With the building of the space charge, the band bending takes place in the semiconductor that allows electrons to tunnel directly into the conduction band from metal through the potential barrier as shown in the schematic diagram in Fig. 4(a). The constituted tunneling current is given by Shockley equation

$$
J=\operatorname{AT}^{2}\left(\exp ^{\frac{-\mathrm{e} \phi_{\mathrm{b}}}{\mathrm{kT}}}\right)\left[\exp ^{\frac{\mathrm{ev}}{\mathrm{e} \text { eff }}}-1\right]
$$

where $k, \phi_{b}$, and $V_{\text {eff }}$ are Boltzman constant, Schottky barrier height, and the potential difference across the semiconductor generated due to the applied field, respectively. At the beginning with lower fields, thermally active electrons are the only source that contributes to the space charge formation, as tunneling probability of electron through the barrier is negligible. Moreover, the Schottky barrier height at interface restricts the emission current and for higher values of $\phi_{b}$, current density reduces further. Here, it is worth mentioning that our $\mathrm{MoS}_{2}$ samples show n-type behavior. ${ }^{44}$ For Pt-MoS (few-layer) interface, the reported barrier height was estimated to be $\sim 0.2 \mathrm{eV}$ (Ref. 20) and expected to increase further for monolayer $\mathrm{MoS}_{2}$ due to opening up of the band gap. This accounts for lowering of the magnitude of emission current observed in monolayer $\mathrm{MoS}_{2}$ at lower applied fields. In

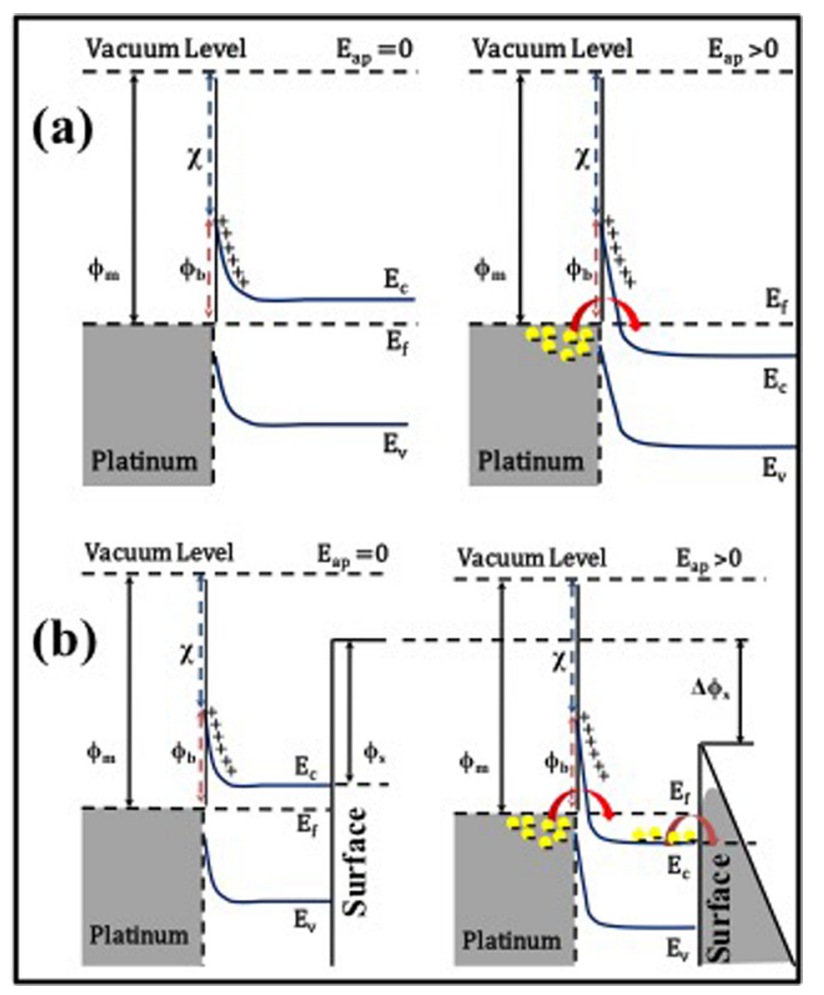

FIG. 4. Schematic of a two step emission mechanism. (a) Band bending at the metal-semiconductor interface in the presence of the applied field, when $\mathrm{E}_{\mathrm{ap}}>0$ electrons from metal tunnel into the conduction band. (b) Surface barrier lowering followed by electron tunneling into the vacuum due to the lowering of the surface barrier shown in gray color. 


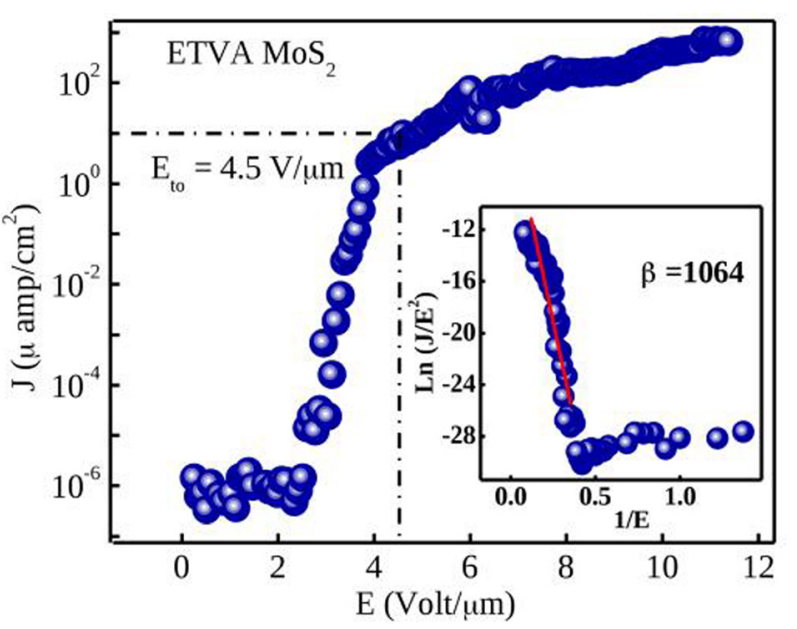

FIG. 5. FE J-V curves of edge exposed $\mathrm{MoS}_{2}$ film, and inset shows the FN plot.

$1 \mathrm{~L}-\mathrm{MoS}_{2}$, other intrinsic factors such as higher effective mass and enhanced interactions among confined charge carriers might also contribute in the reduction of emission current. $^{40,41}$ Hence, the magnitude of semiconductor work function and space charges accumulated inside the semiconducting layer are also significant to achieve low threshold field. For this, we have evaluated the work functions for fewlayers, ETVA, and mono layer $\mathrm{MoS}_{2}$ using KPFM, which will be discussed later. A certain minimum thickness of the semiconductor is indeed necessary for building up space charge inside the layer. The theoretically estimated thickness for planar $\mathrm{TiO}_{2}$ was found to be $\sim 4 \mathrm{~nm}$ and below this thickness increase in the concentration of space charge was found to be field independent. ${ }^{38}$ Similarly, in the case of $1 \mathrm{~L}-\mathrm{MoS}_{2}$ sufficient space charges were not able to be acquired due to ultra low thickness $(0.7 \mathrm{~nm})$, which in turn affected the band bending process. As a result, it requires higher fields for emission with respect to few-layer $\mathrm{MoS}_{2}$. In the second step, simultaneously with band bending, under the effect of applied field, vacuum barrier is lowered due to Schottky effect and electrons begin to emit from the semiconductor surface into the vacuum as shown in the schematic of Fig. 4(b). It may be pointed out that the emission characteristic of planar $\mathrm{MoS}_{2}$ samples is argued assuming the ideal condition surface without considering the surface states, roughness, and foreign contaminants.

Contrary to the planar structure, sharp edge geometry is preferred due to the large confinement of the localized electric field that showed lower turn-on field, with large emission current density. Figure 5 shows the FE behavior of EVTA $\mathrm{MoS}_{2}$ film for which $\mathrm{E}_{\text {to }}$ was found $\sim 4.5 \mathrm{~V} / \mu \mathrm{m}$ with higher numerical values of $\beta$ (1064), extracted from linearly fitted I$\mathrm{V}$ data at a higher electric field utilizing Eq. (2). For the $\beta$ calculation magnitude of work function for edge exposed $\mathrm{MoS}_{2}$ was estimated to be $\sim 4.18 \mathrm{eV}$ using KPFM.

The emission current density showed significant improvement and was found to be $\sim 1 \mathrm{~m}-\mathrm{A} / \mathrm{cm}^{2}$. Similar enhanced FE behavior has been reported for other solids consisting of nano-protrusions. The surface of $\mathrm{MoS}_{2}$ film containing oriented layers has average roughness below $1 \mathrm{~nm}$, which could also be realized by the contrast of the HRTEM image shown in Fig. 1(a), confirming that stripe like grains were not extended beyond the flat surface, thereof, the observed value of $\mathrm{E}_{\text {to }}$ and emission current magnitude might differ from $\mathrm{MoS}_{2}$ nanoflowers to sheets.

Other factors, such as the work function of the vertically aligned $\mathrm{MoS}_{2}$ layers, might be different and, hence, could have significant contributions in emission behavior. To further understand the obtained field emission results for few-layers of planar, monolayer $\mathrm{MoS}_{2}$, and EVTA films in terms of their work functions, KPFM was used to evaluate the work functions. It may be pointed out that KPFM is considered to be a suitable non-contact technique and has been used to map the two-dimensional distributions of contact potential difference $\left(V_{\mathrm{CPD}}\right)$ between the tip and a sample. The $V_{C P D}$ can further be exploited to evaluate the work function $\left(\Phi_{\text {sample }}\right)$ of the material through the following mathematical equation: ${ }^{42}$

$$
\Phi_{\text {sample }}=\Phi_{\text {tip }}-e V_{\mathrm{CPD}}
$$

where $\Phi_{\text {tip }}$ and $e$ are the work function of the AFM tip and electronic charge, respectively. ${ }^{42,43}$ The work function of the tip used in the present study ( $\mathrm{Si}$ coated with $\mathrm{Pt} / \mathrm{Ir}$ ) is $\Phi_{\text {tip }}=4.45 \mathrm{eV}$.

The measured two-dimensional distributions of contact potential difference $\left(V_{\mathrm{CPD}}\right)$ of few-layers of planar, monolayer $\mathrm{MoS}_{2}$, and ETVA films are shown in Figures 6(a)-6(c). Using Eq. (3), the work function of the few-layers of planar, monolayer $\mathrm{MoS}_{2}$, and ETVA films are found to be $\sim 4.39$, 4.82 , and $4.18 \mathrm{eV}$, respectively. This result suggests that the lowest value of work function for ETVA also accounts for its improved FE performance as compared with few-layer and mono layer $\mathrm{MoS}_{2}$ thin films.

Hence, ultra thin ETVA-MoS 2 films have shown cold emission characteristics in the proximity of other carbonbased materials. Moreover, the sulfurization method has adequate space to improve the density of such edge exposed $\mathrm{MoS}_{2}$ films (Ref. 23) to tune the emission behavior further. The field emission stability was monitored over a period of $12 \mathrm{~h}$ for the emission current density at 500 and $150 \mu \mathrm{A} / \mathrm{cm}^{2}$ for ETVA and few-layer $\mathrm{MoS}_{2}$, respectively, and corresponding time-dependent plots have been provided in Ref. 44. Few-layer planar $\mathrm{MoS}_{2}$ film has shown better stability
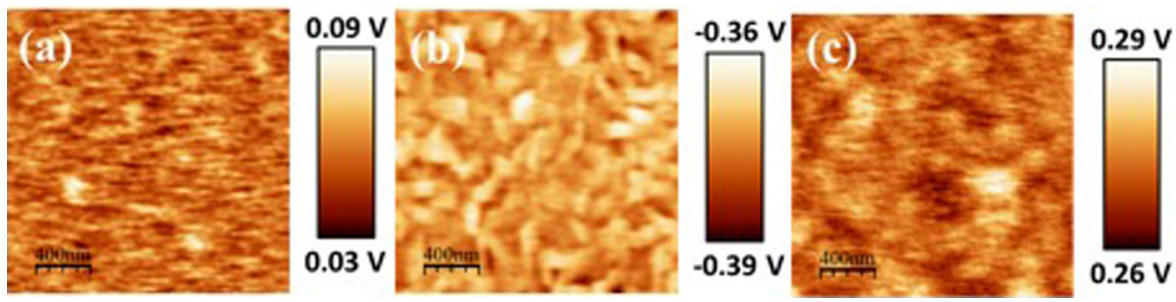

FIG. 6. Two-dimensional distributions of contact potential difference $\left(\mathrm{V}_{\mathrm{CPD}}\right)$ between the tip and (a) few-layers, (b) 1L, and (c) EVTA $\mathrm{MoS}_{2}$ thin films. In all the figures, the scale bar is $400 \mathrm{~nm}$. 
over ETVA $\mathrm{MoS}_{2}$ film. The fluctuation in the ETVA emission current density might be due to the presence of different emission sites at exposed edges.

Ultra thin molybdenum films were sulfurized at two different reaction temperatures to yield few-layer and ETVA $\mathrm{MoS}_{2}$ films, and single layer $\mathrm{MoS}_{2}$ film was synthesized via the CVD method. The thickness of the few-layer film measured $\sim 7-8 \mathrm{~nm}$ while presence of PL spectrum confirmed the monolayer deposition. A HRTEM image of ETVA $\mathrm{MoS}_{2}$ revealed that the nanosize grains consisted of vertically aligned $\mathrm{MoS}_{2}$ layers. A comparative study of cold cathode emission was performed on these few-layer and single layer $\mathrm{MoS}_{2}$ films. The observed emission results affirmed that edge exposed $\mathrm{MoS}_{2}$ exhibited $\mathrm{E}_{\mathrm{to}} \sim 4.5 \mathrm{~V} / \mu \mathrm{m}$ and emission current density $\sim 1 \mathrm{~m}-\mathrm{amp} / \mathrm{cm}^{2}$ at $10 \mathrm{~V} / \mu \mathrm{m}$ and has good field emission stability that can be used in vacuum microelectronic applications. In comparison with other chemical synthesis methods reported so far to fabricate nanostructures of $\mathrm{MoS}_{2}$, the sulfurization method is more efficient to modify surface topography, which in turn exhibits improved emission behavior.

A.P.S.G. and R.S.K. acknowledge the financial support from the DOE (Grant No. DEG02-ER46526). A.M.R. acknowledges the NSF (Grant No. 0841338). We also acknowledge the NSF for financial support (Cooperative Agreement No. EPS-01002410). We thank Mr. D. Barrionuevo for AFM measurements, and Dr. M. Ahmadi and Dr. M. Guinel for TEM work. We thank Dr. T. Som, Institute of Physics, Bhubaneswar, India, for extending his support to carry out the KPFM measurements.

${ }^{1}$ W. B. Choi, D. S. Chung, J. H. Kang, H. Y. Kim, Y. W. Jin, I. T. Han, Y. H. Lee, J. E. Jung, N. S. Lee, G. S. Park, and J. M. Kim, Appl. Phys. Lett. 75(20), 3129 (1999).

${ }^{2}$ K. B. K. Teo, E. Minoux, L. Hudanski, F. Peauger, Jean-P. Schnell, L. Gangloff, P. Legagneux, D. Dieumegard, G. A. J. Amaratunga, and W. I. Milne, Nature 437(7061), 968 (2005).

${ }^{3}$ H. Sugie, M. Tanemura, V. Filip, K. Iwata, K. Takahashi, and F. Okuyama, Appl. Phys. Lett. 78(17), 2578 (2001).

${ }^{4}$ G. Z. Yue, Q. Qiu, B. Gao, Y. Cheng, J. Zhang, H. Shimoda, S. Chang, J. P. Lu, and O. Zhou, Appl. Phys. Lett. 81(2), 355 (2002).

${ }^{5}$ W. A. de Heer, A. Châtelain, and D. Ugarte, Science 270(5239), 1179 (1995).

${ }^{6}$ C. A. Spindt, C. E. Holland, A. Rosengreen, and I. Brodie, IEEE Trans. Electron Devices 38(10), 2355 (1991).

${ }^{7}$ K. B. K. Teo, M. Chhowalla, G. A. J. Amaratunga, W. I. Milne, P. Legagneux, G. Pirio, L. Gangloff, D. Pribat, V. Semet, V. T. Binh, W. H. Bruenger, J. Eichholz, H. Hanssen, D. Friedrich, S. B. Lee, D. G. Hasko, and H. Ahmed, J. Vac. Sci. Technol., B 21(2), 693 (2003).

${ }^{8}$ S. T. Purcell, P. Vincent, C. Journet, and V. T. Binh, Phys. Rev. Lett. 88(10), 105502 (2002).

${ }^{9}$ X. Xu, C. Tang, H. Zeng, T. Zhai, S. Zhang, H. Zhao, Y. Bando, and D. Golberg, ACS Appl. Mater. Interfaces 3(4), 1352 (2011).

${ }^{10}$ Cheng-K. Huang, Y. Ou, Y. Bie, Q. Zhao, and D. Yu, Appl. Phys. Lett. 98(26), 263104 (2011).
${ }^{11}$ D. R. Shinde, P. G. Chavan, S. Sen, D. S. Joag, M. A. More, S. C. Gadkari, and S. K. Gupta, ACS Appl. Mater. Interfaces 3(12), 4730 (2011).

${ }^{12}$ A. Datta, D. Mukherjee, M. Hordagoda, S. Witanachchi, P. Mukherjee, R. V. Kashid, M. A. More, D. S. Joag, and P. G. Chavan, ACS Appl. Mater. Interfaces 5(13), 6261 (2013).

${ }^{13}$ L. Wang, C. Li, Y. Yang, S. Chen, F. Gao, G. Wei, and W. Yang, ACS Appl. Mater. Interfaces 7(1), 526 (2015).

${ }^{14}$ A. K. Geim, Science 324(5934), 1530 (2009).

${ }^{15}$ C. Lee, X. Wei, J. W. Kysar, and J. Hone, Science 321(5887), 385 (2008).

${ }^{16}$ A. Malesevic, R. Kemps, A. Vanhulsel, M. P. Chowdhury, A. Volodin, and C. V. Haesendonck, J. Appl. Phys. 104(8), 084301 (2008).

${ }^{17}$ S. Santandrea, F. Giubileo, V. Grossi, S. Santucci, M. Passacantando, T. Schroeder, G. Lupina, and A. D. Bartolomeo, Appl. Phys. Lett. 98(16), 163109 (2011).

${ }^{18}$ U. A. Palnitkar, R. V. Kashid, M. A. More, D. S. Joag, L. S. Panchakarla, and C. N. R. Rao, Appl. Phys. Lett. 97(6), 063102 (2010).

${ }^{19}$ B. Radisavljevic, A. Radenovic, J. Brivio, V. Giacometti, and A. Kis, Nat. Nanotechnol. 6(3), 147 (2011).

${ }^{20}$ S. Das, Hong-Y. Chen, A. V. Penumatcha, and J. Appenzeller, Nano Lett. 13(1), 100 (2013).

${ }^{21}$ M. Chhowalla and G. A. J. Amaratunga, Nature 407(6801), 164 (2000).

${ }^{22}$ Y. Li, H. Wang, L. Xie, Y. Liang, G. Hong, and H. Dai, J. Am. Chem. Soc. 133(19), 7296 (2011).

${ }^{23}$ D. Kong, H. Wang, J. J. Cha, M. Pasta, K. J. Koski, J. Yao, and Y. Cui, Nano Lett. 13(3), 1341 (2013).

${ }^{24}$ A. P. S. Gaur, S. Sahoo, M. Ahmadi, M. J. F. Guinel, S. K. Gupta, R. Pandey, S. K. Dey, and R. S. Katiyar, J. Phys. Chem. C 117(49), 26262 (2013).

${ }^{25}$ A. P. S. Gaur, S. Sahoo, M. Ahmadi, S. P. Dash, M. J. F. Guinel, and R. S. Katiyar, Nano Lett. 14(8), 4314 (2014).

${ }^{26}$ Yi-H. Lee, L. Yu, H. Wang, W. Fang, X. Ling, Y. Shi, Cheng-T. Lin, JingK. Huang, Mu-T. Chang, Chia-S. Chang, M. Dresselhaus, T. Palacios, Lain-J. Li, and J. Kong, Nano Lett. 13(4), 1852 (2013).

${ }^{27}$ G. Morell, A. González-Berríos, B. R. Weiner, and S. Gupta, J. Mater. Sci. - Mater. Electron. 17(6), 443 (2006).

${ }^{28}$ R. V. Kashid, D. J. Late, S. S. Chou, Yi-K. Huang, M. De, D. S. Joag, M. A. More, and V. P. Dravid, Small 9(16), 2730 (2013).

${ }^{29}$ Y. B. Li, Y. Bando, and D. Golberg, Appl. Phys. Lett. 82(12), 1962 (2003).

${ }^{30}$ D. Varshney, A. V. Sumant, O. Resto, F. Mendoza, K. P. Quintero, M. Ahmadi, B. R. Weiner, and G. Morell, Carbon 63, 253 (2013).

${ }^{31}$ C. Lee, H. Yan, L. E. Brus, T. F. Heinz, J. Hone, and S. Ryu, ACS Nano 4(5), 2695 (2010).

${ }^{32}$ S. Sahoo, A. P. S. Gaur, M. Ahmadi, M. J. F. Guinel, and R. S. Katiyar, J. Phys. Chem. C 117(17), 9042 (2013).

${ }^{33}$ B. Chakraborty, A. Bera, D. V. S. Muthu, S. Bhowmick, U. V. Waghmare, and A. K. Sood, Phys. Rev. B 85(16), 161403 (2012).

${ }^{34}$ I. H. Campbell and P. M. Fauchet, Solid State Commun. 58(10), 739 (1986).

${ }^{35}$ P. Parayanthal and F. H. Pollak, Phys. Rev. Lett. 52(20), 1822 (1984).

${ }^{36}$ A. Splendiani, L. Sun, Y. Zhang, T. Li, J. Kim, Chi-Y. Chim, G. Galli, and F. Wang, Nano Lett. 10(4), 1271 (2010).

${ }^{37}$ R. Stratton, Phys. Rev. 125(1), 67 (1962).

${ }^{38}$ V. T. Binh and C. Adessi, Phys. Rev. Lett. 85(4), 864 (2000).

${ }^{39}$ V. T. Binh, J. P. Dupin, P. Thevenard, S. T. Purcell, and V. Semet, J. Vac. Sci. Technol., B 18(2), 956 (2000).

${ }^{40}$ M. W. Geis, N. N. Efremow, K. E. Krohn, J. C. Twichell, T. M. Lyszczarz, R. Kalish, J. A. Greer, and M. D. Tabat, Nature 393(6684), 431 (1998).

${ }^{41}$ A. González-Berríos, F. Piazza, and G. Morell, J. Vac. Sci. Technol., B 23(2), 645 (2005).

${ }^{42}$ C. Maragliano, S. Lilliu, M. S. Dahlem, M. Chiesa, T. Souier, and M. Stefancich, Sci. Rep. 4, 4203 (2014).

${ }^{43}$ M. Kumar, T. Basu, and T. Som, J. Appl. Phys. 118, 055102 (2015).

${ }^{44}$ See supplementary material at http://dx.doi.org/10.1063/1.4940306 for AFM, TEM, field effect transistor (FET) characteristics, and field emission stability results. 